\title{
A Comparative Study on Multiwavelet Construction Methods and Customized Multiwavelet Library for Mechanical Fault Detection
}

\author{
Jing Yuan, ${ }^{1,2}$ Chenjun Wei, ${ }^{1}$ Bo Zou, ${ }^{1}$ Jun Zhu, ${ }^{1}$ Ying Wei, ${ }^{1}$ Yanyang Zi, ${ }^{2}$ and Yu Zhou ${ }^{1}$ \\ ${ }^{1}$ Shanghai Radio Equipment Institute, Shanghai 200090, China \\ ${ }^{2}$ State Key Laboratory for Manufacturing Systems Engineering, Xian Jiaotong University, Xian 710049, China \\ Correspondence should be addressed to Jing Yuan; yuanjing_802@163.com
}

Received 28 February 2015; Accepted 11 June 2015

Academic Editor: Changjun Zheng

Copyright (C) 2015 Jing Yuan et al. This is an open access article distributed under the Creative Commons Attribution License, which permits unrestricted use, distribution, and reproduction in any medium, provided the original work is properly cited.

Inner product transform principle reveals that the basis functions most relevant or similar to the fault features are pivotal to the meaningful fault detection. Customized multiwavelet methods and practices have continued to improve over the recent years, focused on two-scale similarity transform (TST), lifting transform (LT), and lifting scheme (LS). Due to the respective advantages and disadvantages, a comparative study on the multiwavelet construction methods by TST, symmetric and dissymmetric LT, and LS is discussed in the paper, covering the differences of construction theories, the synthetic analyses of construction strategies, and the comparison of waveform characteristics along with their applicable occasions. Comprehensively utilizing the capabilities of the construction methods, a novel customized multiwavelet library is established for the accurate fault detection. The proposed method is applied to incipient fault detection of rolling bearing for electric locomotive to verify the effectiveness and feasibility.

\section{Introduction}

Operating in long-term and/or complex severe conditions, kernel components and important structures of key mechanical equipment would inevitably generate various faults or damage, yielding unscheduled downtime and costly breakdowns. Therefore, mechanical fault detection has received considerable attention over the recent decades. Such mechanical fault detection methods of linear transform as Fourier transform and wavelet transform $[1,2]$ are revealed as the inner product transform based on Hilbert space [3].

As a typical inner product transform, the emerging multiwavelets are the new development of wavelet theory, attracting remarkable interest of researchers [4-8]. The particular superiority on multiwavelets is as follows. (1) They can possess the important signal processing properties of orthogonality, symmetry, short support, and vanishing moments simultaneously, which traditional scalar wavelets fail to do [9]. (2) Multiwavelets could offer multiple basis functions different in the time-frequency characteristics potential and promising for the weak and multiple feature extraction.
Multiwavelet transform is realized essentially by an inner product operation $\left\langle x(t), \Psi_{a, b}(t)\right\rangle$ of a given signal $x(t)$ and the vector-valued basis functions $\Psi_{a, b}(t)$. Therein, the basis functions $\Psi_{a, b}(t)$ most relevant or similar to the fault features are pivotal to the meaningful fault detection [3]. Thus, customized multiwavelet methods and practices have continued to improve over the recent years, in which two-scale similarity transform (TST) [10], lifting transform (LT) [11], and lifting scheme (LS) [12] have been focused on (note that LT and LS are defined distinctly). The key TST matrix was constructed for custom design of basis functions and applied to fault diagnosis of rolling bearing and rub-impact fault of flue gas turbine unit [13]. Symmetric LT strategy was proposed to construct customized biorthogonal multiwavelets flexibly for fault detection of air compressor [14]. Based on cubic Hermite multiwavelets, signal-adapted multiwavelets with various vanishing moments were established by LTs and utilized to diagnose gearbox fault of rolling mill [15]. Similar to second generation wavelets, various vector prediction and update operators were designed by Hermite spline interpolation for 
customized LS-based multiwavelets and applied to gear crack detection of electric locomotive [16].

Despite the prior progress for customized multiwavelets, such challenges as the appropriate selection of multiwavelet construction methods for a given fault detection in engineering practice still remain. To overcome the problem, a study on the aforementioned multiwavelet construction methods is discussed in the paper. The advantages and disadvantages of these construction theories are first discussed. Then, the synthetic analyses of construction strategies are investigated. Besides, the characteristics of each customized multiwavelet by TST, symmetric and dissymmetric LT, and LS are summarized along with their applicable occasions. Furthermore, an extensive customized multiwavelet library by these construction methods is set up for the accurate analysis of mechanical fault detection. The proposed multiwavelet library is applied to the incipient fault detection of rolling bearing for electric locomotive.

The remainder of this paper is organized as follows. A brief introduction of multiwavelet theory and the aforementioned multiwavelet construction methods are reviewed in Section 2. Their contrastive study is given in Section 3. A customized multiwavelet library is addressed and validated in Section 4. Section 5 provides conclusions.

\section{Summary of Multiwavelets and Construction Methods}

2.1. Multiwavelet Theory. By two-scale equations, multiwavelet decomposition is addressed by

$$
\begin{aligned}
\mathbf{c}_{j-1, n} & =\sum_{k \in Z} \mathbf{H}_{k-2 n} \mathbf{c}_{j, k}, \\
\mathbf{d}_{j-1, n} & =\sum_{k \in Z} \mathbf{G}_{k-2 n} \mathbf{c}_{j, k},
\end{aligned}
$$

where $\mathbf{c}_{j-1, n}$ and $\mathbf{d}_{j-1, n}$ are the low-frequency and highfrequency coefficients, respectively, and $\left\{\mathbf{H}_{k}\right\}$ and $\left\{\mathbf{G}_{k}\right\}$ are the matrix low-pass and high-pass filter coefficients, respectively. And multiwavelet reconstruction is obtained by

$$
\mathbf{c}_{j, k}=\sum_{n} \mathbf{H}_{k-2 n}^{*} \mathbf{c}_{j-1, n}+\sum_{n} \mathbf{G}_{k-2 n}^{*} \mathbf{d}_{j-1, n} .
$$

The superscript $*$ stands for the complex conjugate transpose.

Due to the initial expansion coefficients, preprocessing must be conducted in advance, in which the oversampling representation performs well for feature extraction and is adopted in the paper [17]. Besides, the postprocessing is canceled in the paper to extract the diverse fault feature based on the inner product transform principle [15].

2.2. Two-Scale Similarity Transform. TST proposed by Strela [17] can be used to transfer approximation order back and forth between multiple scaling functions and their duals. Suppose that $\mathbf{M}(\omega)$ is a well-defined TST matrix in which $\mathbf{H}(0)$ and $\mathbf{M}(0)$ share a common right eigenvector r. Let $\boldsymbol{\Phi}$, $\boldsymbol{\Psi}$ and $\widetilde{\boldsymbol{\Phi}}, \widetilde{\boldsymbol{\Psi}}$ be biorthogonal multiwavelets with the symbols of $\mathbf{H}(\omega), \mathbf{G}(\omega), \widetilde{\mathbf{H}}(\omega)$, and $\widetilde{\mathbf{G}}(\omega)$. Therein, $\boldsymbol{\Phi}$ and $\widetilde{\boldsymbol{\Phi}}$ have the approximation orders $p$ and $\widetilde{p} \geq 1$, respectively. TST is described by [17]

$$
\begin{aligned}
& \mathbf{H}_{\text {new }}(\omega)=\frac{1}{2} \mathbf{M}(2 \omega) \mathbf{H}(\omega) \mathbf{M}^{-1}(\omega), \\
& \mathbf{G}_{\text {new }}(\omega)=\frac{1}{2} \mathbf{G}(\omega) \mathbf{M}^{-1}(\omega), \\
& \widetilde{\mathbf{H}}_{\text {new }}(\omega)=\mathbf{2} \mathbf{M}^{-*}(2 \omega) \widetilde{\mathbf{H}}(\omega) \mathbf{M}^{*}(\omega), \\
& \widetilde{\mathbf{G}}_{\text {new }}(\omega)=\mathbf{2} \widetilde{\mathbf{G}}(\omega) \mathbf{M}^{*}(\omega)
\end{aligned}
$$

By TST, $\boldsymbol{\Phi}_{\text {new }}$ has the approximation order $p+1$ and $\widetilde{\boldsymbol{\Phi}}_{\text {new }}$ has the approximation order $\tilde{p}-1$. Besides, another new biorthogonal pair is formed by (3). The calculation of $\mathbf{M}(\omega)$ is proved to be the key for multiwavelet construction.

2.3. Lifting Transform. Taking initial multiwavelets, LT successively modifies the multiple wavelet functions and finetunes such properties as the numbers of vanishing moments. Denote $\boldsymbol{\Phi}, \boldsymbol{\Psi}$ and $\widetilde{\boldsymbol{\Phi}}, \widetilde{\boldsymbol{\Psi}}$ as compactly supported biorthogonal multiwavelets with the polyphase symbols of $\mathbf{H}(z), \mathbf{G}(z)$, $\widetilde{\mathbf{H}}(z)$, and $\widetilde{\mathbf{G}}(z)$. The multiwavelet LT is addressed by [11]

$$
\begin{aligned}
& \mathbf{H}_{\text {new }}(z)=\mathbf{H}(z) \\
& \mathbf{G}_{\text {new }}(z)=\mathbf{T}\left(z^{2}\right)\left(\mathbf{G}(z)+\mathbf{S}\left(z^{2}\right) \mathbf{H}(z)\right), \\
& \widetilde{\mathbf{H}}_{\text {new }}(z)=\widetilde{\mathbf{H}}(z)-\mathbf{S}^{*}\left(z^{2}\right) \widetilde{\mathbf{G}}(z), \\
& \widetilde{\mathbf{G}}_{\text {new }}(z)=\left(\mathbf{T}^{*}\left(z^{2}\right)\right)^{-1} \widetilde{\mathbf{G}}(z) .
\end{aligned}
$$

Here, $\mathbf{S}(z)$ and $\mathbf{T}(z)$ are finite degree, which play the vital role in multiwavelet construction, and the determinant of $\mathbf{T}(z)$ is a monomial. The new biorthogonal family shares the same $\Phi$ with the initiating one. Besides, LT raises the vanishing moments of $\boldsymbol{\Psi}$. In particular, LT mixes $\boldsymbol{\Phi}$ and $\boldsymbol{\Psi}$, which makes $\Psi_{\text {new }}$ flexible and multivariate.

2.4. Lifting Scheme. LS is a powerful construction tool for biorthogonal multiwavelets derived in the spatial domain. Starting with the vector input signal f, LS consists of the split, predict, and update steps. The decomposition and reconstruction of multiwavelet LS are illustrated in Figure 1, where $\mathbf{P}$ and $\mathbf{U}$, respectively, represent the vector prediction and update operators, significant for multiwavelet construction. Furthermore, the biorthogonal perfect reconstruction multifilter banks can also be given by (5) through $z$ transforms [12]:

$$
\begin{aligned}
& \mathbf{G}(z)=z\left(\mathbf{I}-\frac{\mathbf{P}\left(z^{2}\right)}{z}\right), \\
& \mathbf{H}(z)=\mathbf{I}+\mathbf{U}\left(z^{2}\right) \mathbf{G}(z), \\
& \widetilde{\mathbf{H}}(z)=\mathbf{I}+\frac{\mathbf{P}\left(z^{2}\right)}{z}, \\
& \widetilde{\mathbf{G}}(z)=\frac{1}{z}\left(\mathbf{I}-\mathbf{H}(z) z \mathbf{U}\left(z^{2}\right)\right) .
\end{aligned}
$$




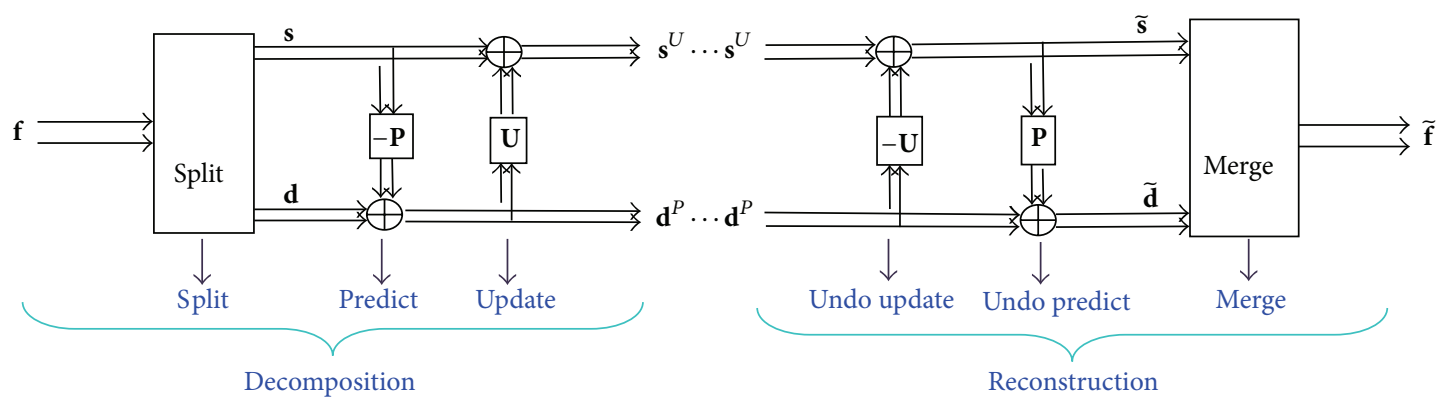

FIgURE 1: Multiwavelet LS.

\section{A Contrastive Study on the Multiwavelet Construction Methods}

\subsection{Discussion of Construction Theory}

3.1.1. Two-Scale Similarity Transform. TST is a new nonobvious construction method for customized multiwavelets. Taking an existing multiwavelet system as an origin, a series of new changeable biorthogonal multiwavelets could be obtained by TST. Practically, the approximation order and regularity of multiple scaling functions are raised in the TST, with such good properties as finite support and symmetry of multiwavelet system remaining uncharged. However, the approximation order and regularity of the dual multiple scaling functions are decreased in TST, which makes the unbalance of basis functions and their dual ones. Hence, we could not simultaneously construct the basis functions of good properties along with their dual ones. Moreover, TST constructs new multiple scaling and wavelet functions only, respectively, using the corresponding original multiple scaling and wavelet functions, which could not sharply change the waveforms of basis functions. Furthermore, the TST approach involves the operation of matrix division or singular matrices, resulting in the complex and slow computation.

3.1.2. Lifting Transform. On the basis of the perfect reconstruction filter bands, biorthogonal multiwavelets are obtained by designing the lifting coefficients in LT, modifying the characteristic of the original multiwavelets. Without the matrix division, LT is simple, fast, and flexible for construction. In LT, the vanishing moments of multiple wavelet functions are modified with the symmetry or dissymmetry to satisfy the requirement of different signals. New multiple wavelet functions by LT are actually the linear combination of the original multiple scaling and wavelet functions, which could result in the large change of the waveform. Moreover, LT always produces the short finite support of basis functions, due to the flexible constrain of the support length. In spite of the advantages, LT only constructs the new multiple wavelet functions, without any changes of the multiple scaling functions, leading to the same decomposition coefficients of the lowest frequency band. Furthermore, the dual multiple scaling and wavelet functions show the low regularity, reducing the precision of signal reconstruction. Thus, the signal decomposition using LT-based customized multiwavelets is often applied in fault detection, without signal reconstruction.

3.1.3. Lifting Scheme. Similar to second generation wavelets, LS-based customized multiwavelets derived in the spatial domain do not rely on Fourier transform. LS has the advantages of simple structured design, flexible adaptive structure, high speed calculation, and less occupied memory. In LS, the multiwavelet construction turns out to be the design of the vector prediction and update operators, in which the vanishing moments could be designed for a given signal. Such properties as finite support, regularity, and symmetry could be preserved in LS. However, the main peaks of waveforms of diverse basis functions corresponding to the designed vector prediction and update operators are very analogous. Different free parameter in LS only changes the oscillation number and waveform of the subpeaks of basis functions.

\subsection{Analysis of Construction Strategy}

3.2.1. TST-Based Customized Multiwavelets. TST modifying the approximation order shows a nonobvious construction for customized multiwavelets to detect rotating machinery faults. Starting with GHM multiwavelets [18], a TST is first performed with $\mathbf{M}_{1}(\omega)$ shown in (6) to construct a series of changeable biorthogonal multiwavelets with the good properties of symmetry, regularity, and finite support. Moreover, another TST with $\mathbf{M}_{2}(\omega)$ shown in (7) is carried out to the dual multiwavelets $\widetilde{\mathbf{H}}_{\text {new }}(\omega)$ and $\widetilde{\mathbf{G}}_{\text {new }}(\omega)$ to improve the properties of the dual ones [13]:

$$
\begin{aligned}
& \mathbf{M}_{1}(\omega)=\left[\begin{array}{cc}
a\left(1+e^{-i \omega}\right) & -2 \sqrt{2} a \\
b\left(1-e^{-i \omega}\right) & 0
\end{array}\right], \\
& \mathbf{M}_{2}(\omega)=\left[\begin{array}{cc}
c & 0 \\
d\left(1+e^{-i \omega}\right) & e\left(1-e^{-i \omega}\right)
\end{array}\right] .
\end{aligned}
$$

Here, $[a, b, c, d, e]$ are nonzero parameters, which influence the customized multiwavelets together.

Based on the kurtosis maximization principle, the construction strategy for TST-based customized multiwavelets is shown in Figure 2. 


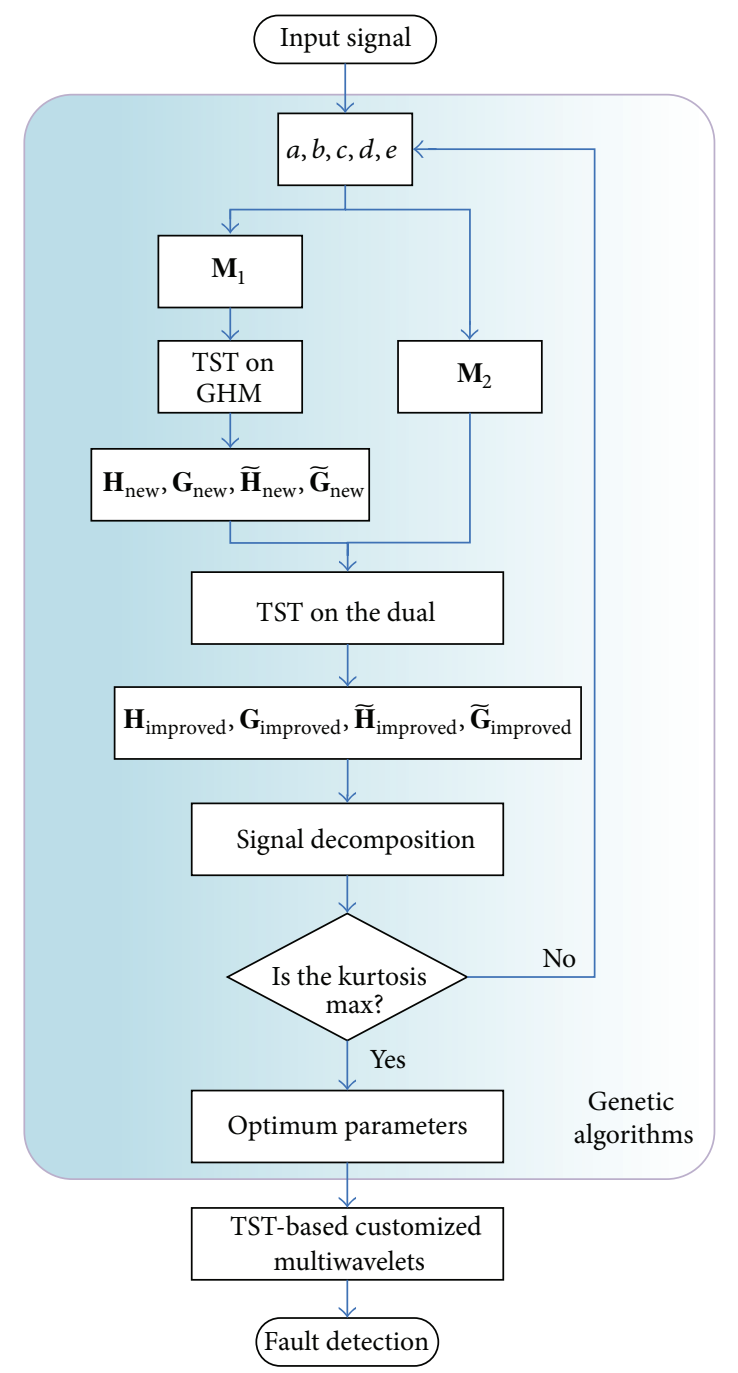

FIGURE 2: Construction strategy for TST-based customized multiwavelets.

3.2.2. LT-Based Customized Multiwavelets with Various Vanishing Moments. In fault detection, basis functions should have enough vanishing moments such that singularity signatures can be extracted from the noisy signals. Hence, LT is adopted to construct a series of biorthogonal multiwavelets of regularity and short support with various vanishing moments. Note that, because the localized faults often generate the dynamic impulsive response characterized as the unilateral oscillating decay, the symmetry of basis functions is not deliberately constrained in the construction method based on the inner product transform principle. On the basis of the fixed cubic Hermite multiwavelets [19], the critical S( $z)$ in LT from 1st to 7th vanishing moments are listed as follows [15]:

$$
\begin{aligned}
& \text { 1st: } \mathbf{S}_{1}=\left[\begin{array}{cc}
2.776 \times 10^{-17} & a_{1} \\
-0.694 \times 10^{-17} & b_{1}
\end{array}\right] \text {, } \\
& \text { 2nd: } \mathbf{S}_{2}=\left[\begin{array}{cc}
0.028 & 1.219 \\
-0.007 & -0.208
\end{array}\right] \times 10^{-15} \text {, } \\
& \text { 3rd: } S_{3}=\left[\begin{array}{cccc}
-2.8916 \times 10^{-16}+0.0935 a_{3} & 0.9833+0.7009 a_{3} & 1.0364 \times 10^{-16}-0.0935 a_{3} & -0.9833+0.7009 a_{3} \\
6.7571 \times 10^{-18}+0.0935 b_{3} & -7.3689 \times 10^{-16}+0.7009 b_{3} & -1.3696 \times 10^{-17}-0.0935 b_{3} & 7.3416 e \times 10^{-16}+0.7009 b_{3}
\end{array}\right], \\
& \text { 4th: } \mathbf{S}_{4}=\left[\begin{array}{cccc}
0 & 0.9833 & 0 & -0.9833 \\
-0.1996 & -1.4969 & 0.1996 & -1.4969
\end{array}\right] \text {, } \\
& \text { 5th: } \mathbf{S}_{5}=\left[\begin{array}{cccccc}
0.1249+0.0488 a_{5} & 1.8599+0.2720 a_{5} & -0.1875 & -0.3963+0.9205 a_{5} & 0.0626-0.0488 a_{5} & -0.5297+0.2720 a_{5} \\
-0.1120+0.488 b_{5} & -1.0088+0.2720 b_{5} & 0.1996 & 0.1551+0.9205 b_{5} & -0.0876-0.0488 b_{5} & 0.4881+0.2720 b_{5}
\end{array}\right] \text {, }
\end{aligned}
$$


6th: $\mathbf{S}_{6}=\left[\begin{array}{cccccc}0.4629 & 3.7431 & -0.1875 & 5.9776 & -0.2754 & 1.3535 \\ -0.2133 & -1.5731 & 0.1996 & -1.7547 & 0.0137 & -0.0762\end{array}\right]$

7th: $\mathbf{S}_{7}$

$=\left[\begin{array}{cccccccc}0.4053+0.0198 a_{7} & 3.4939+0.0973 a_{7} & -0.4958+0.0467 a_{7} & 2.8178+0.6985 a_{7} & -0.0697-0.0467 a_{7} & -3.1685+0.6985 a_{7} & 0.1601-0.0198 a_{7} & -0.8205+0.0973 a_{7} \\ -0.1918+0.0198 b_{7} & -1.4723+0.0973 b_{7} & 0.2731+0.0467 b_{7} & -0.8484+0.6985 b_{7} & -0.0462-0.0467 b_{7} & 1.0118+0.6985 b_{7} & -0.0351-0.0198 b_{7} & 0.1769+0.0973 b_{7}\end{array}\right]$.

Here, free parameters $a_{i}, i=1,3,5,7$, affect $\mathbf{S}_{i}$ with odd vanishing moments on the first row and $b_{i}, i=1,3,5,7$, on the second row. Meanwhile, there is only one multiwavelet pair at each even level. Furthermore, $\mathbf{T}(z)$ is also ignored (i.e., $\mathbf{T}(z)=\mathbf{I})$ because it has no effect on the multiple wavelet basis functions along with their vanishing moments.

Using the well-defined local spectral entropy minimization rule for the typical mechanical faults [15], the construction strategy for LT-based customized multiwavelets with various vanishing moments is illustrated in Figure 3, in which the appropriate vanishing moments are also selected to capture the hidden fault features effectively.

3.2.3. Symmetric LT-Based Customized Multiwavelets. As one of the important properties for multiwavelets, symmetry could ensure the property of linear phase or at least general linear phase of the multifilters, effectively avoiding reconstruction errors. Therefore, the symmetric selection is adopted in LT to obtain the symmetric or antisymmetric basis functions. If $\omega_{0}(x)$ is employed as an origin to construct a new wavelet with the specified numbers of vanishing moments, then LT of (4) is equal to

$$
\omega_{0}^{\text {new }}=\omega_{0}(x)+\sum_{i=1}^{k} c_{i} \omega_{i}(x)
$$

where $c_{i}$ is the lifting coefficient. Lifting vanishing moments $p$ of $\Psi$ up to $p^{\prime}$, integrate both sides of (9); then the symmetric selection for LT is obtained by [14]

$$
\left[\begin{array}{ccc}
\int \omega_{1}\left(x+k_{\omega_{1}, 1}\right) x^{p} d x & \int \omega_{1}\left(x+k_{\omega_{1}, 2}\right) x^{p} d x & \cdots \\
\int \omega_{1}\left(x+k_{\omega_{1}, 1}\right) x^{p+1} d x & \int \omega_{1}\left(x+k_{\omega_{1}, 2}\right) x^{p+1} d x & \cdots \\
\vdots & \vdots \\
\vdots & & \vdots \\
\int \omega_{1}\left(x+k_{\omega_{1}, 1}\right) x^{p^{\prime}-1} d x & \int \omega_{1}\left(x+k_{\omega_{1}, 2}\right) x^{p^{\prime}-1} d x & \cdots
\end{array}\right]\left[\begin{array}{cc}
1 & \\
B_{\omega_{0}} B_{\omega_{1}} & \ddots \\
0 &
\end{array}\right]\left[\begin{array}{c}
c_{1} \\
c_{2} \\
\vdots \\
c_{k}
\end{array}\right]=\left[\begin{array}{c}
-\int \omega_{0}(x) x^{p} d x \\
-\int \omega_{0}(x) x^{p+1} d x \\
\vdots \\
-\int \omega_{0}(x) x^{p^{\prime}-1} d x
\end{array}\right],
$$

where $k$ is the translation quantity of basis functions and $B_{\omega_{i}}= \pm 1$ represents the symmetry and antisymmetry of basis functions.

By the kurtosis maximization principle, the construction strategy for symmetric LT-based customized multiwavelets is shown in Figure 4.

3.2.4. LS-Based Customized Multiwavelets. LS has an inherent tool for custom design of multiwavelets for mechanical fault detection. Based on Hermite spline interpolation, a series of variable LS-based multiwavelets with the properties of biorthogonality, symmetry, short support, and vanishing moments are constructed. The vector prediction operator $\mathbf{P}$ and vector update operator $\mathbf{U}$ satisfy [16]

$$
\begin{aligned}
\mathbf{P} & =\{P(0), P(-1)\}, \\
P(0) & =\left(\begin{array}{cc}
\frac{1}{2} & \frac{1}{4} \\
c & -\frac{1}{4}
\end{array}\right),
\end{aligned}
$$

$$
P(-1)=\left(\begin{array}{cc}
\frac{1}{2} & -\frac{1}{4} \\
-c & -\frac{1}{4}
\end{array}\right)
$$$$
\mathbf{U}=\{U(0), U(1)\},
$$$$
U(0)=\left(\begin{array}{rr}
\frac{1}{4} & -\frac{1}{8} \\
-\frac{c}{2} & -\frac{1}{8}
\end{array}\right) \text {, }
$$$$
U(1)=\left(\begin{array}{cc}
\frac{1}{4} & \frac{1}{8} \\
\frac{c}{2} & -\frac{1}{8}
\end{array}\right) \text {, }
$$

where $c$ is the free parameter.

Using the entropy minimization rule, the construction strategy for LS-based customized multiwavelets is illustrated in Figure 5. 


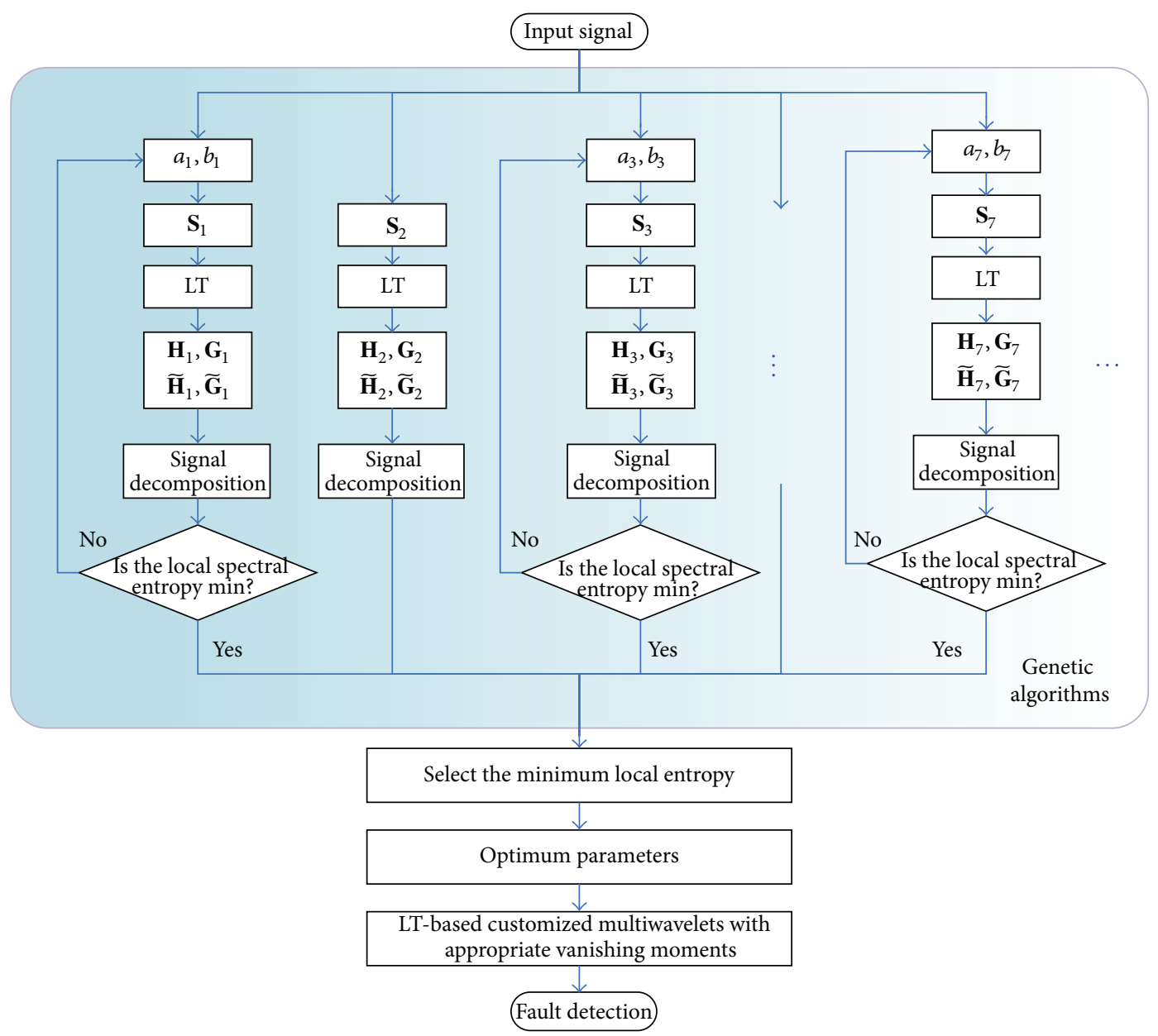

FIGURE 3: Construction strategy for LT-based customized multiwavelets with various vanishing moments.

\subsection{Comparison of Waveform Characteristic and Applicable Occasion}

3.3.1. TST-Based Customized Multiwavelets. Based on GHM multiwavelets, a series of TST-based customized multiwavelets are constructed. One improved multiwavelet example with $a=-1, b=-2, c=4, d=6$, and $e=-9$, implementing the procedure of Figure 2, is shown in Figure 6. The multiwavelets show an impact characteristic waveform of compact support and quick oscillating decay. The main peaks of the TST-based multiwavelets are sharp, similar to the fault features of impact faults or friction faults. Thus, the TST-based customized multiwavelets are quite suitable for the impact and friction fault detection, for instance, the rubimpact fault of flue gas turbine unit in [13].

3.3.2. LT-Based Customized Multiwavelets with Various Vanishing Moments. Taking cubic Hermite multiwavelets as an origin, a family of LT-based customized multiwavelets with various vanishing moments is obtained according to Figure 3. Figure 7 displays multiple wavelet functions of vanishing moments from 1 to 7 , with $a_{i}=2$ and $b_{i}=1, i=$ $1,3,5,7$. It could be seen from Figure 7 that the amount of unilateral oscillating decay is enhanced with the increase of the vanishing moments. Meanwhile, the waveforms of $\psi_{1}$ change a little within the low vanishing moments, shown in (a) (d) in Figure 7. Nevertheless, the waveforms of $\psi_{2}$ change variously. The multiwavelet family presents unilateral oscillating decay, potential of optimally matching the dynamic impulsive response of mechanical localized faults based on the inner product transform principle. Hence, LT-based customized multiwavelets with various vanishing moments could be adopted for mechanical localized fault diagnosis such as the slight scratch fault of rolling bearing in [15].

3.3.3. Symmetric LT-Based Customized Multiwavelets. Introducing the symmetric selection into LT, symmetric multiple wavelet functions are designed following the flowchart of Figure 4. One example of the symmetric LT-based multiple wavelet functions with $\mathbf{c}=\left[\begin{array}{cccc}-0.2 & -0.2 & 0.1 & -0.5 \\ 1 & 0.5 & 0.1 & 0.3\end{array}\right]$ is illustrated in Figure 8. Compared with the LT-based multiwavelets with various vanishing moments in Figure 7, some of the wavelet functions are similar due to the same origin basis functions and construction theory. However, the symmetric family is not more changeable than the dissymmetric one. Like the 


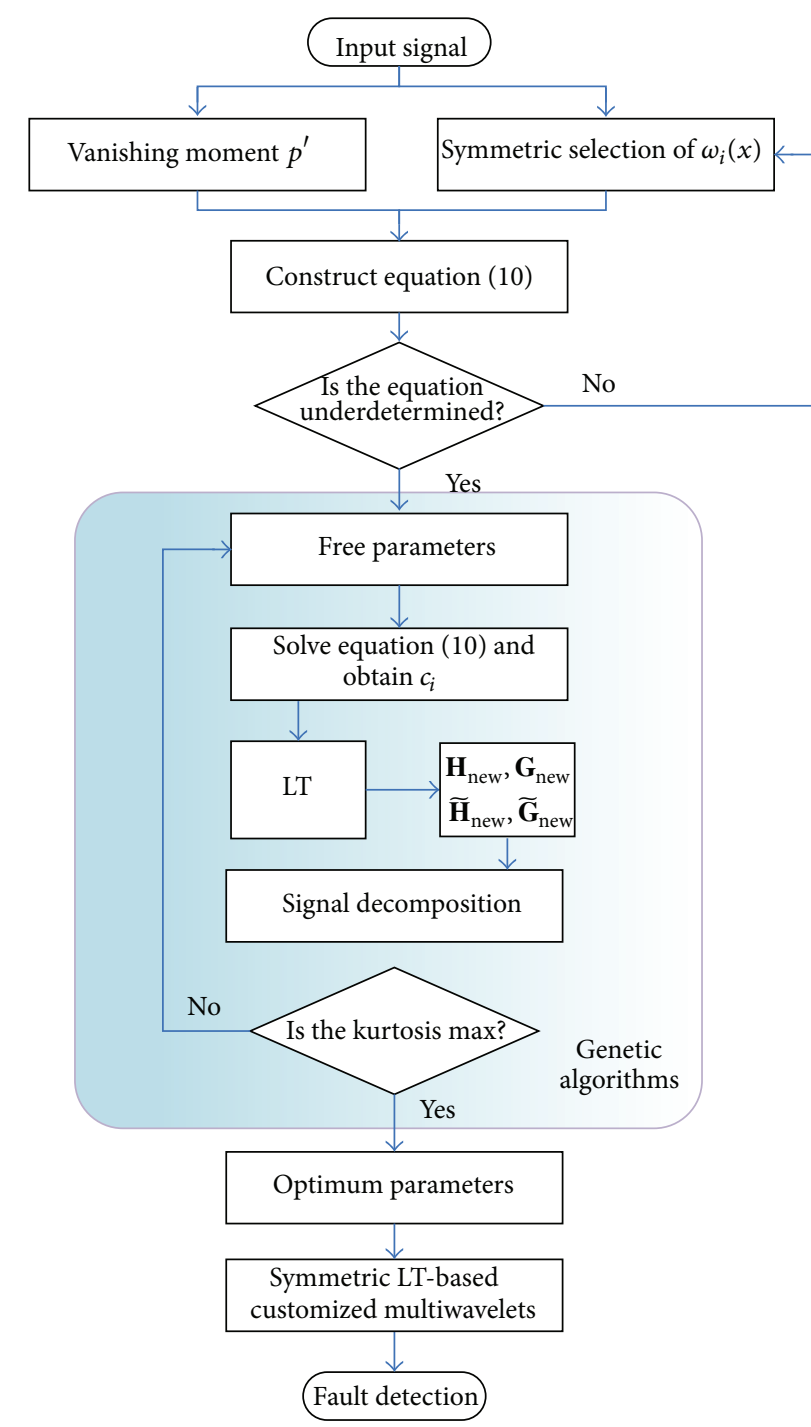

FIgURE 4: Construction strategy for symmetric LT-based customized multiwavelets.

LT-based customized multiwavelets with various vanishing moments, symmetric LT-based customized multiwavelets are also appropriate for mechanical localized fault diagnosis. Importantly, the symmetric restriction of multiwavelets brings benefits to avoiding reconstruction error of signal processing. Hence, if the signal processing of fault detection requires high quality, the symmetric LT-based customized multiwavelets are more suitable than the dissymmetric ones.

3.3.4. LS-Based Customized Multiwavelets. On the basis of Hermite spline interpolation, the vector prediction and update operators are, respectively, designed according to Figure 5. One example of LS-based multiwavelets corresponding to the vector prediction operator and vector update operator with $c=-1$ is shown in Figure 9. The basis functions perform as the impulsive characteristic of quick oscillating decay with the main peak dominating in the finite support, similar to the fault feature response of the large damping

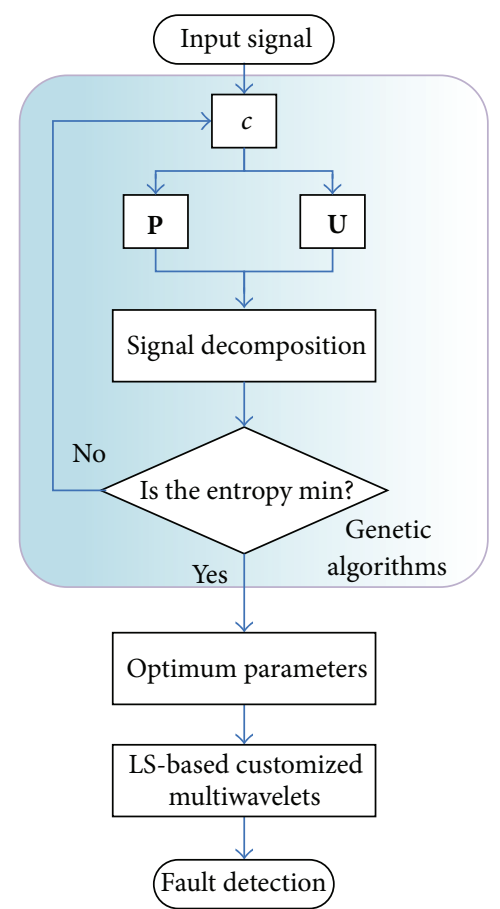

FIGURE 5: Construction strategy for LS-based customized multiwavelets.

system. Hence, the LS-based customized multiwavelets are befitting for capturing mechanical faults in the large damping system such as rolling mills or electric locomotives in [16].

3.4. Summary. In conclusion, different construction theories and strategies have their respective advantages and disadvantages. Meanwhile, the above four types of customized multiwavelets are distinctive in the waveform characteristics. Thus, an appropriate multiwavelet construction method is selected according to the properties and waveforms of basis functions in the custom design of multiwavelets in engineering practice. Therein, the most similar or relative multiwavelets are constructed and selected according to a given analyzed signal based on the inner product transform principle for the meaningful and effective mechanical fault detection.

\section{A Customized Multiwavelet Library and Validation}

4.1. A Customized Multiwavelet Library. Considering the various advantages and disadvantages among the aforementioned construction theories and strategies along with the multiwavelet characteristics, an extensive customized multiwavelet library is established by TST, symmetric and dissymmetric LT, and LS for the accurate analysis of mechanical fault detection. Figure 10 shows the strategy of customized multiwavelet library, in which the selection rule could be the kurtosis maximization, the entropy minimization, and so on.

4.2. Experimental Validation. The proposed customized multiwavelet library is validated for bearing fault detection on 

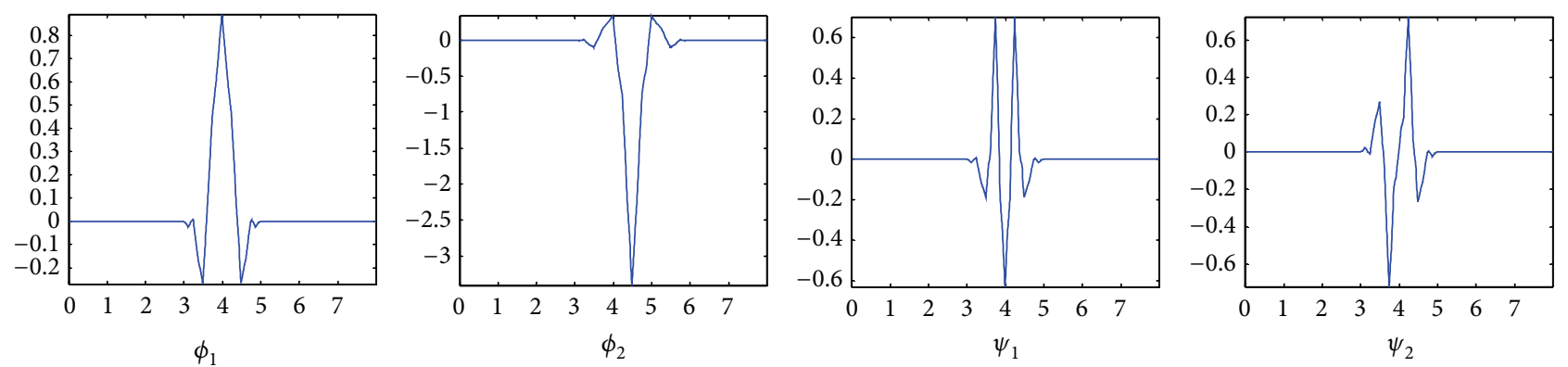

(a)
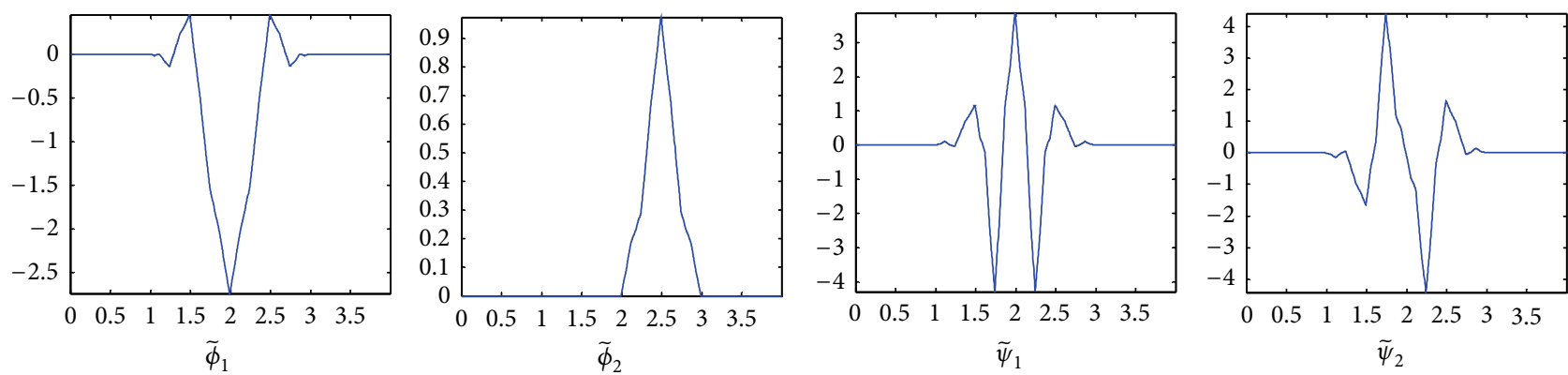

(b)

FIGURE 6: TST-based multiwavelets: (a) multiple scaling and wavelet functions; (b) dual multiple scaling and wavelet functions.

TABLE 1: The geometric parameters of the tested bearing.

\begin{tabular}{lc}
\hline Parameter & Value \\
\hline Bearing specs & $552732 \mathrm{QT}$ \\
Inner race diameter & $160 \mathrm{~mm}$ \\
Outer race diameter & $290 \mathrm{~mm}$ \\
Roller diameter & $34 \mathrm{~mm}$ \\
Roller number & 17 \\
Contact angle & $0^{\circ}$ \\
\hline
\end{tabular}

electric locomotive test facility shown in Figure 11. The locomotive was jacked up and the shafts were idling at a speed of $650 \mathrm{r} / \mathrm{min}$. Vibration signals were collected by accelerometers magnetically attached on the bearing housing by a sampling frequency $12.8 \mathrm{kHz}$. The geometric parameters of test bearing are listed in Table 1. The characteristic frequency of outer race fault for the bearing is calculated at $78.169 \mathrm{~Hz}$, that is, $0.0128 \mathrm{~s}$.

One noisy signal is displayed in Figure 12, which does not provide any warning of bearing fault. We apply the proposed customized multiwavelet library to analyze the data. The entropy minimization of spectral envelope is recommended to select the optimal multiwavelets, by measuring the sparsity of the spectral envelope [15]. Considering the abundant information, the high-frequency coefficients at the three-level decomposition are subjected to the entropy minimization rule. According to the strategy of Figure 10, the signal-adapted multiwavelets are the LT-based multiwavelets dominated by $a_{1}=39.4933$ and $b_{1}=62.6189$ with vanishing moment 1, shown in Figure 13. The purified high-frequency result at the three-level decomposition on the first channel by the optimal multiwavelets is illustrated in Figure 14. Evenly spaced impulses exist in the result, whose inverse is about $0.0128 \mathrm{~s}$, coinciding with the characteristic frequency of outer race fault. This implies that there is a localized fault on the outer race of the test bearing.

In order to affirm the effectiveness of the proposed method, it is also compared with the TST-based and symmetric LT-based and LS-based customized multiwavelets, whose purified results are displayed in Figure 15. We see from Figure 15 that only part of the impulsive sequence is extracted by the symmetric LT-based method, along with the meaningless disorder signals acquired by the other two methods. Thus, the contrastive results could hardly provide information to the diagnostic conclusion. After being disassembled, a slight rub was found on the outer race of the test bearing shown in Figure 16, consistent with the diagnostic conclusion of the proposed method. Moreover, the experimental case coincides with the applicable occasion of each customized multiwavelet family proposed in Section 3.3.

\section{Conclusions}

Owing to the respective advantages and disadvantages of the obvious multiwavelet construction methods, customized multiwavelets for mechanical fault detection suffer from such main challenges as the appropriate construction approach. Thus, a study on the TST, symmetric and dissymmetric LT, and LS multiwavelet construction methods is discussed in the paper, covering the differences of construction theories, the synthetic analyses of construction strategies, and the 

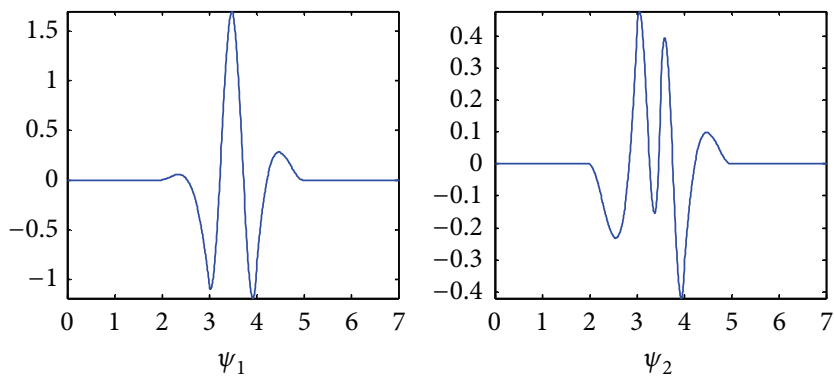

(a)
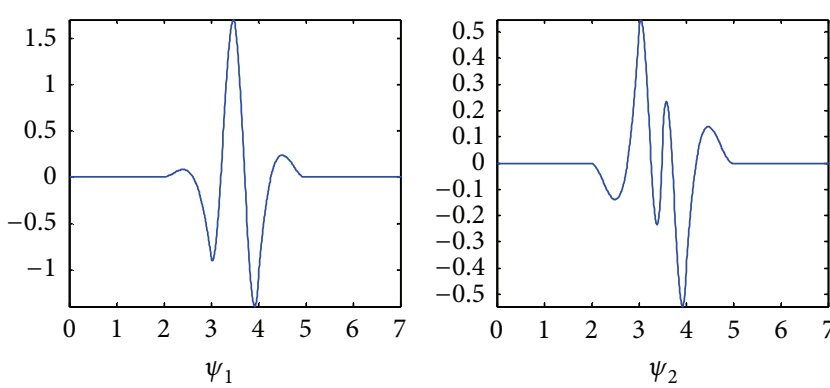

(c)
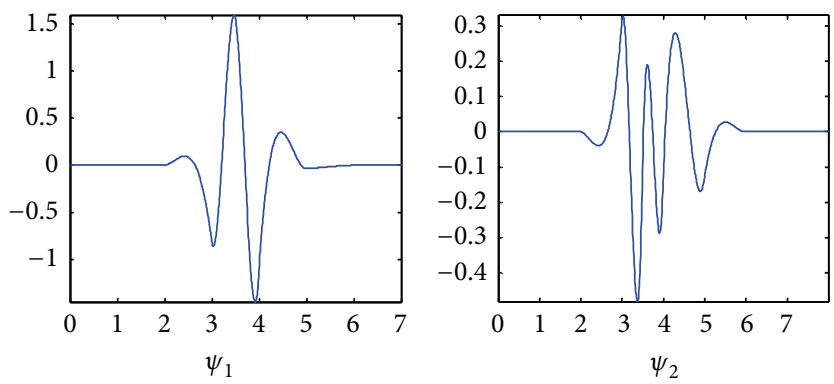

(e)

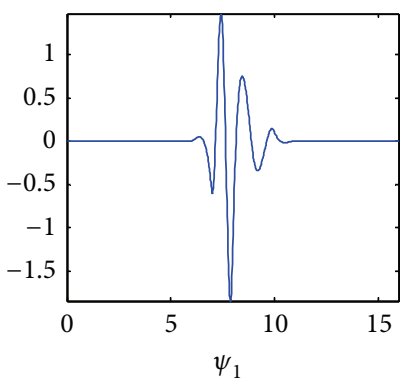

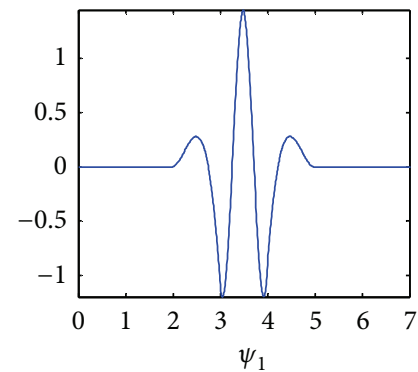

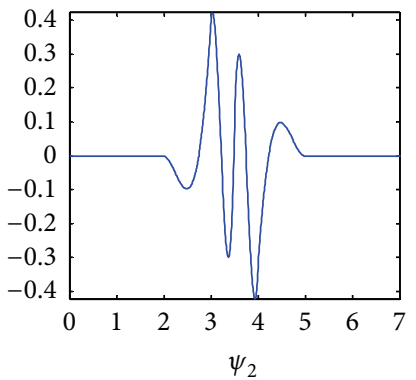

(b)
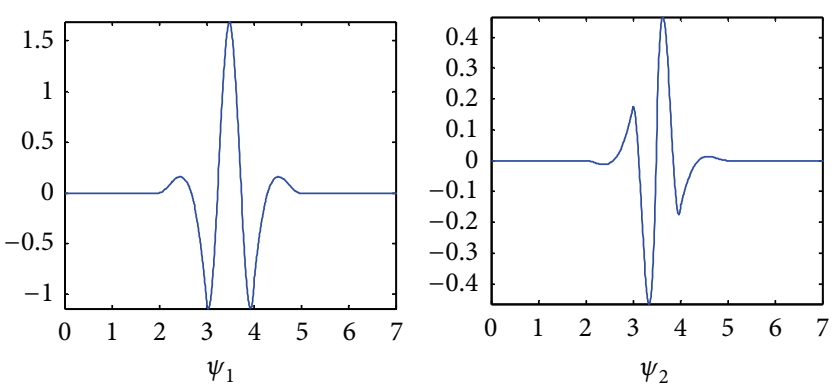

(d)
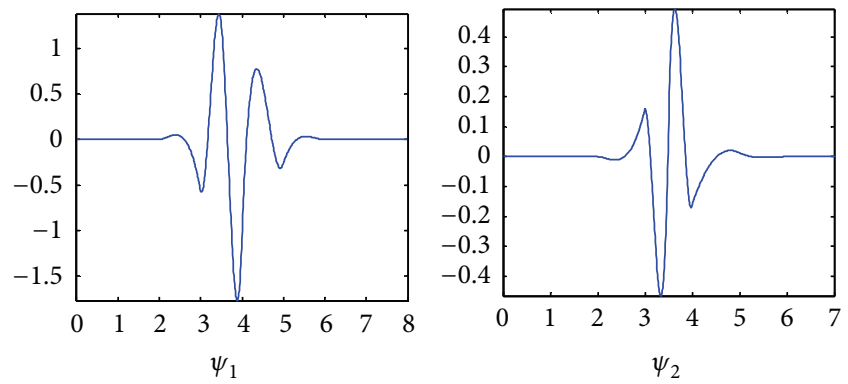

(f)

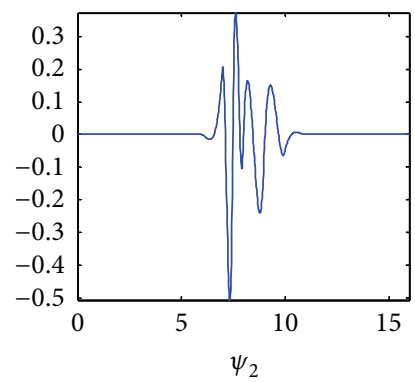

$(\mathrm{g})$

FIGURE 7: LT-based multiple wavelet functions with various vanishing moments: (a) 1st; (b) 2nd; (c) 3rd; (d) 4th; (e) 5th; (f) 6th; (g) 7th.

comparisons of waveform characteristics along with their applicable occasions. In engineering practice, the simple and straightforward selection approach for an appropriate multiwavelet construction method is as follows.

(1) TST-based customized multiwavelets are quite suitable for the impact and friction fault detection, for instance, the rub-impact fault of large mechanical unit.
(2) LT-based customized multiwavelets with various vanishing moments could be adopted for mechanical localized fault diagnosis such as the slight scratch fault of rolling bearing.

(3) Symmetric LT-based customized multiwavelets are also appropriate for mechanical localized fault diagnosis, especially in the case of the high requirement of signal processing. 

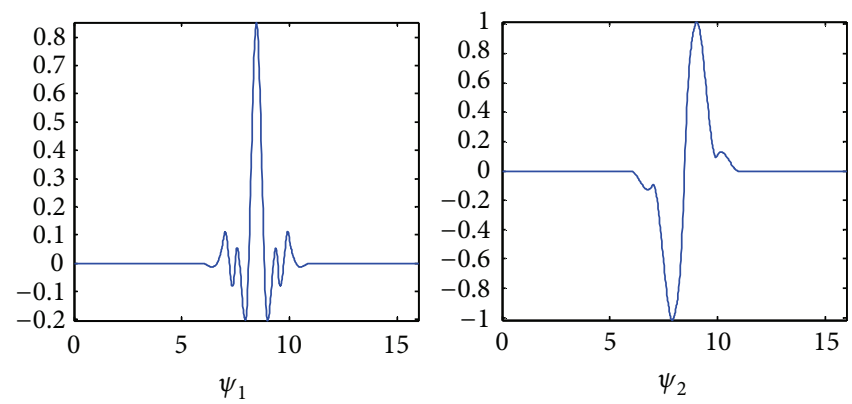

Figure 8: Symmetric LT-based multiwavelets.
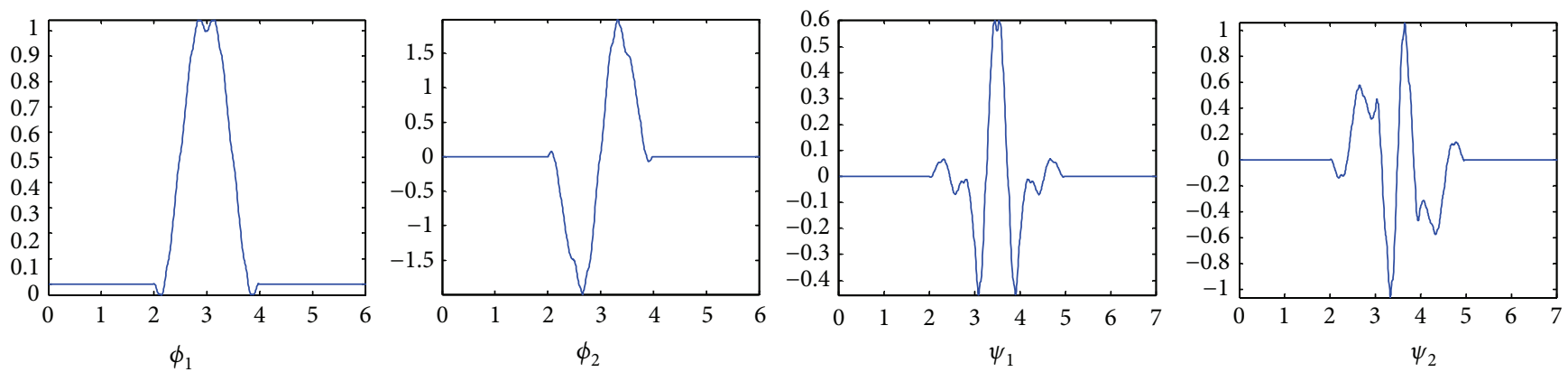

FIGURE 9: LS-based multiwavelets.

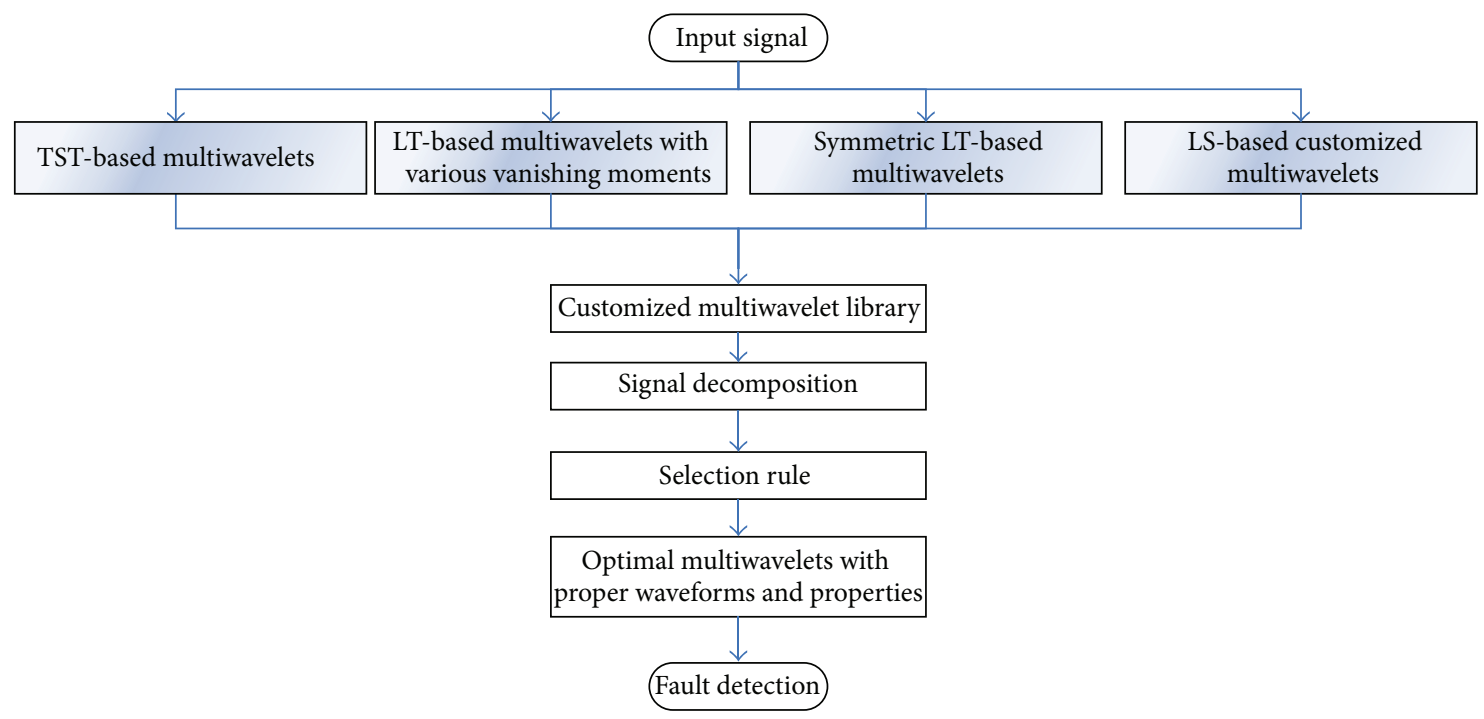

FIGURE 10: Construction strategy of customized multiwavelet library.

(4) LS-based customized multiwavelets are befitting for capturing mechanical faults in the large damping system such as rolling mills or electric locomotives.

Comprehensively utilizing the capabilities of the construction methods, a novel customized multiwavelet library is established for the more effective fault detection than that of each single method. The experimental validation of the proposed library is conducted on incipient bearing fault diagnosis for electric locomotive to verify the effectiveness and feasibility.

\section{Conflict of Interests}

The authors declare that there is no conflict of interests regarding the publication of this paper.

\section{Acknowledgments}

The paper is commemorated to Professor Zhengjia He, who unfortunately died of cancer. Sincere thanks are due for his instruction and enlightenment, which the authors 


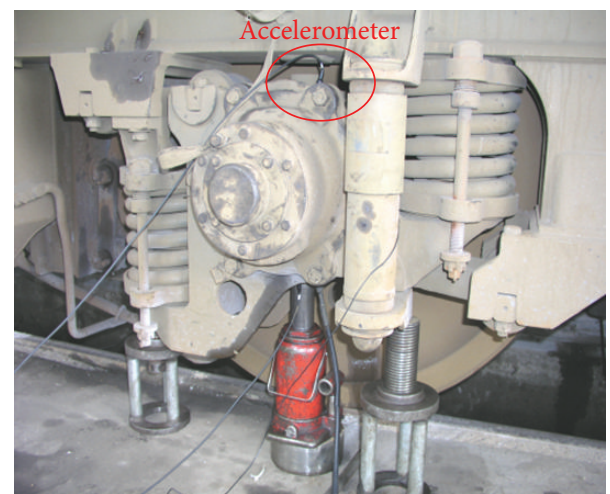

FIGURE 11: Electric locomotive test facility.

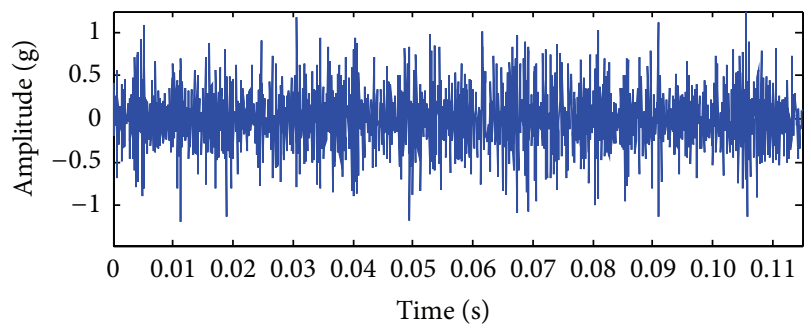

FIGURE 12: The analyzed signal.
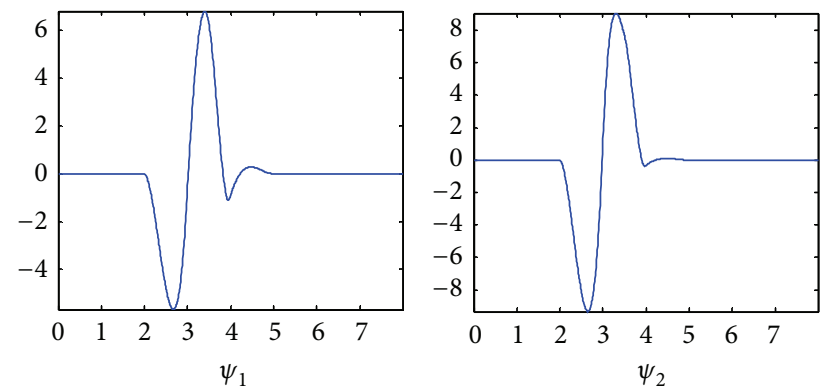

FIgURE 13: The customized multiple wavelet functions.

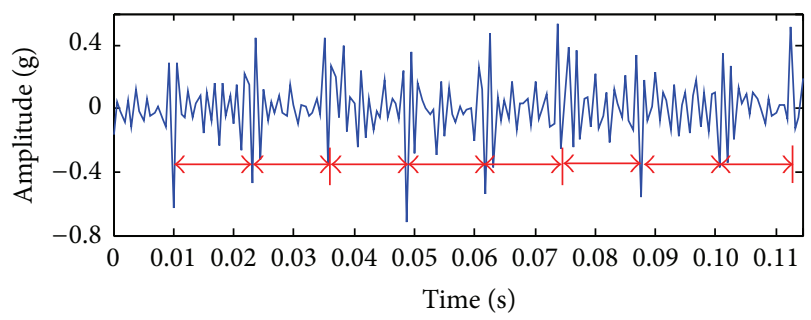

FIGURE 14: The purified result using the proposed method.

can benefit from in their whole lives. This work is supported by National Nature Science Foundation of China (no. 51405301) and Shanghai Science and Technology Committee (no. 13111101300). Besides, the authors gratefully thank Dr. Xiaodong Wang for his contributions to the research.

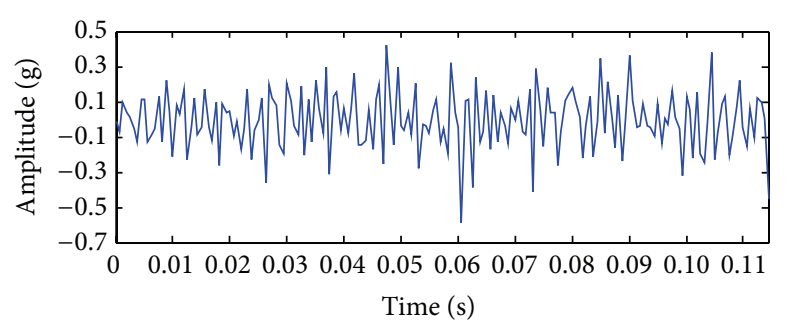

(a)

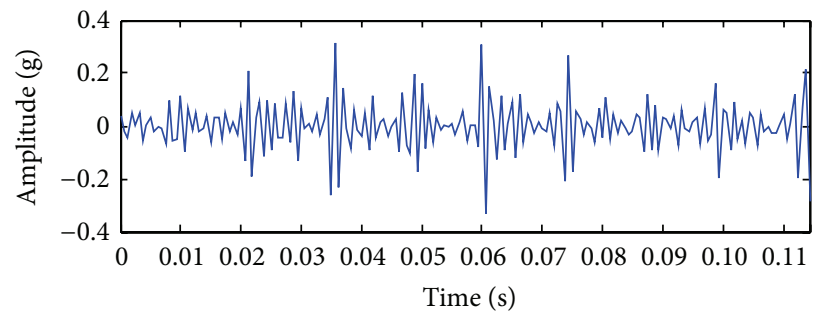

(b)

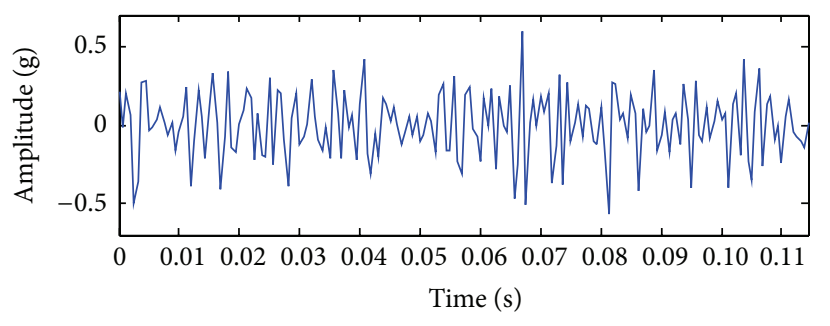

(c)

FIGURE 15: The purified results using the compared methods: (a) by TST-based multiwavelets; (b) by symmetric LT-based multiwavelets; (c) by LS-based multiwavelets.

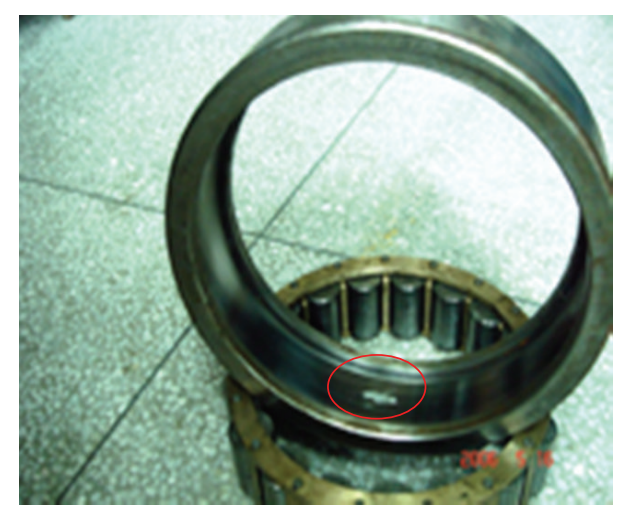

FIGURE 16: Slight rub fault on the outer race of the testing bearing.

\section{References}

[1] J. Xiang, Z. Jiang, and X. Chen, "A class of wavelet-based Rayleigh-Euler beam element for analyzing rotating shafts," Shock and Vibration, vol. 18, no. 3, pp. 447-458, 2011.

[2] C.-W. Fei and G.-C. Bai, "Wavelet correlation feature scale entropy and fuzzy support vector machine approach for aeroengine whole-body vibration fault diagnosis," Shock and Vibration, vol. 20, no. 2, pp. 341-349, 2013. 
[3] Z. J. He, H. R. Cao, Z. Li, Y. Zi, and X. Chen, "The principle of second generation wavelet for milling cutter breakage detection," Science in China Series E: Technological Sciences, vol. 52, no. 5, pp. 1312-1322, 2009.

[4] A. Z. Averbuch, V. A. Zheludev, and T. Cohen, "Multiwavelet frames in signal space originated from Hermite splines," IEEE Transactions on Signal Processing, vol. 55, no. 3, pp. 797-808, 2007.

[5] Y. M. Wang, X. F. Chen, and Z. J. He, "An adaptive inverse iteration algorithm using interpolating multiwavelets for structural eigenvalue problems," Mechanical Systems and Signal Processing, vol. 25, no. 2, pp. 591-600, 2011.

[6] C. Zhao, Y. Gao, J. He, and J. Lian, "Recognition of driving postures by multiwavelet transform and multilayer perceptron classifier," Engineering Applications of Artificial Intelligence, vol. 25, no. 8, pp. 1677-1686, 2012.

[7] Y. Wang, G. Ji, P. Lin, and E. Trucco, "Retinal vessel segmentation using multiwavelet kernels and multiscale hierarchical decomposition," Pattern Recognition, vol. 46, no. 8, pp. 21172133, 2013.

[8] H. K. Jiang, C. L. Li, and H. X. Li, "An improved EEMD with multiwavelet packet for rotating machinery multi-fault diagnosis," Mechanical Systems and Signal Processing, vol. 36, no. 2, pp. 225-239, 2013.

[9] I. Daubechies, Ten Lectures of Wavelets, CBMS-NSF Regional Conference Series in Applied Mathematics, Society for Industrial and Applied Mathematics, Philadelphia, Pa, USA, 1992.

[10] V. Strela, "Multiwavelets: regularity, orthogonality, and symmetry via two-scale similarity transform," Studies in Applied Mathematics, vol. 98, no. 4, pp. 335-354, 1997.

[11] F. Keinert, "Raising multiwavelet approximation order through lifting," SIAM Journal on Mathematical Analysis, vol. 32, no. 5, pp. 1032-1049, 2001.

[12] A. Z. Averbuch and V. A. Zheludev, "Lifting scheme for biorthogonal multiwavelets originated from Hermite splines," IEEE Transactions on Signal Processing, vol. 50, no. 3, pp. 487-500, 2002.

[13] J. Yuan, Z. J. He, Y. Y. Zi, Y. Lei, and Z. Li, "Adaptive multiwavelets via two-scale similarity transforms for rotating machinery fault diagnosis," Mechanical Systems and Signal Processing, vol. 23, no. 5, pp. 1490-1508, 2009.

[14] X. D. Wang, Y. Y. Zi, and Z. J. He, "Multiwavelet construction via an adaptive symmetric lifting scheme and its applications for rotating machinery fault diagnosis," Measurement Science and Technology, vol. 20, no. 4, Article ID 045103, 2009.

[15] J. Yuan, Z. J. He, Y. Y. Zi, and Y. Wei, "Construction and selection of lifting-based multiwavelets for mechanical fault detection," Mechanical Systems and Signal Processing, vol. 40, no. 2, pp. 571588, 2013.

[16] J. Yuan, Z. He, and Y. Zi, "Gear fault detection using customized multiwavelet lifting schemes," Mechanical Systems and Signal Processing, vol. 24, no. 5, pp. 1509-1528, 2010.

[17] V. Strela, Multiwavelets: Theory and Application, Massachusetts Institute of Technology, Cambridge, Mass, USA, 1996.

[18] J. S. Geronimo, D. P. Hardin, and P. R. Massopust, "Fractal functions and wavelet expansions based on several scaling functions," Journal of Approximation Theory, vol. 78, no. 3, pp. 373-401, 1994.

[19] W. Dahmen, B. Han, R.-Q. Jia, and A. Kunoth, "Biorthogonal multiwavelets on the interval: cubic Hermite splines," Constructive Approximation, vol. 16, no. 2, pp. 221-259, 2000. 

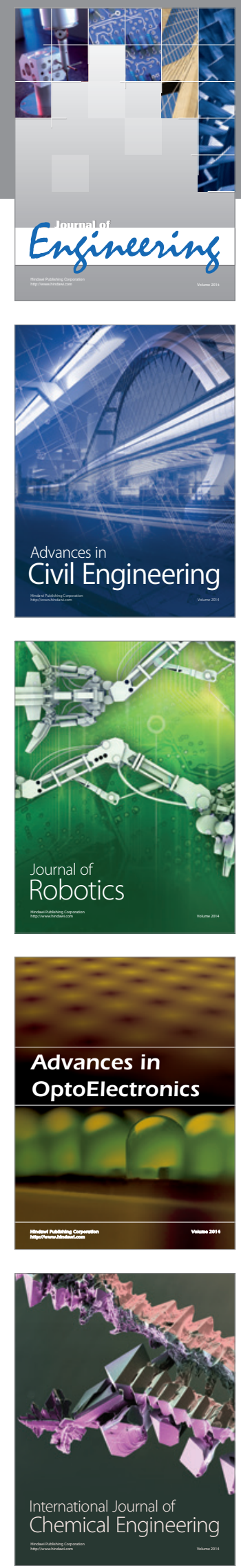

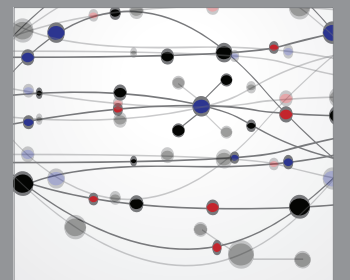

The Scientific World Journal
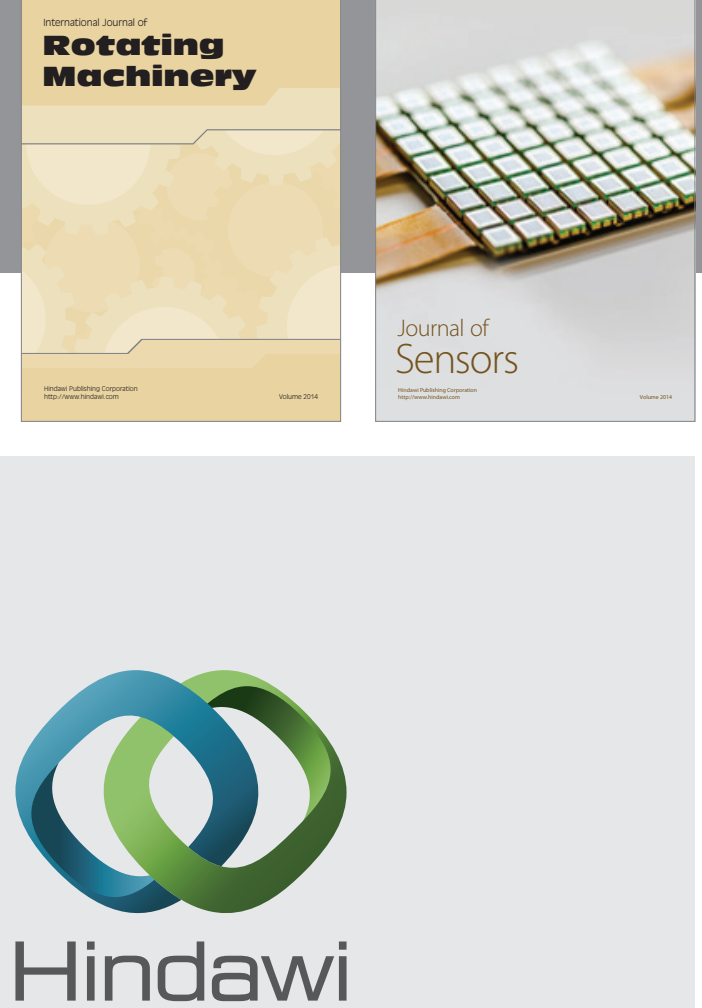

Submit your manuscripts at http://www.hindawi.com
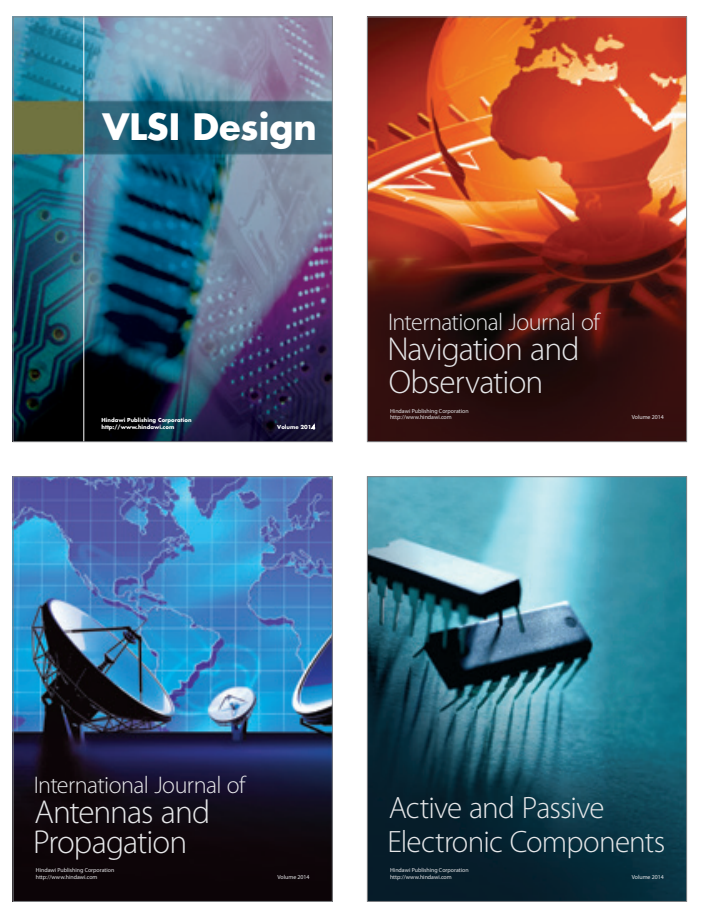
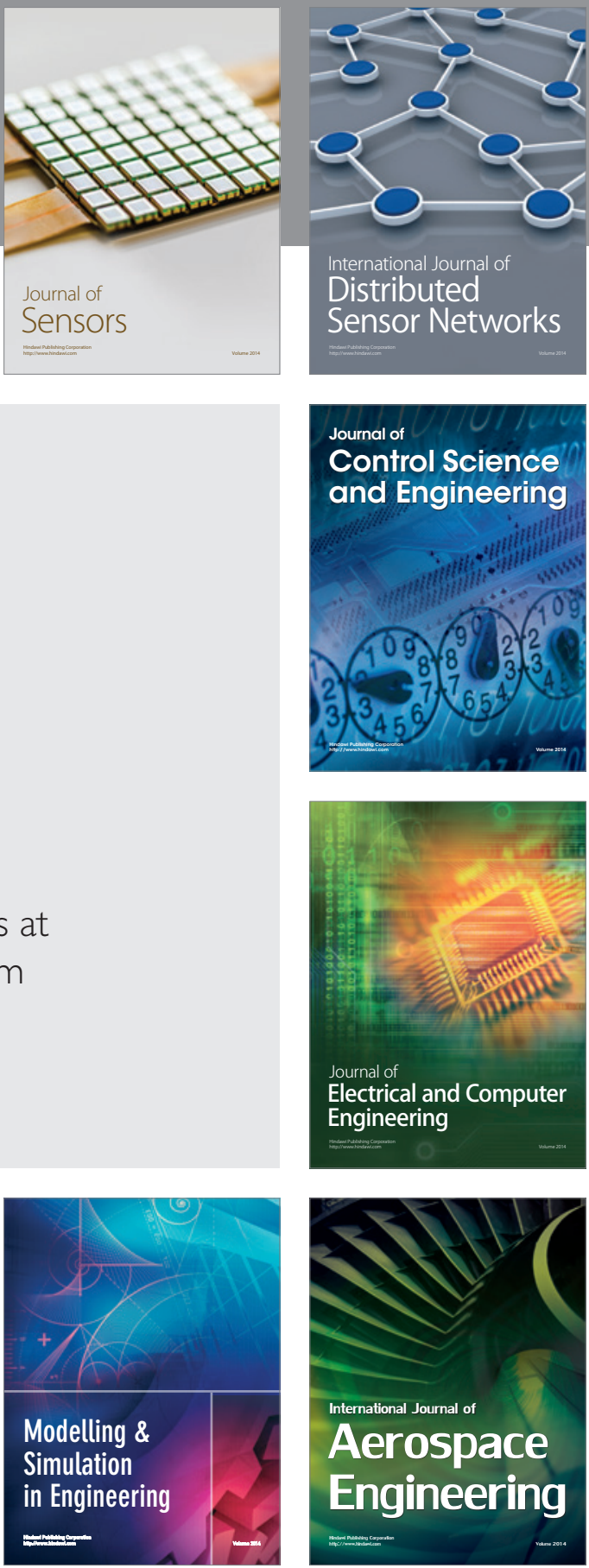

Journal of

Control Science

and Engineering
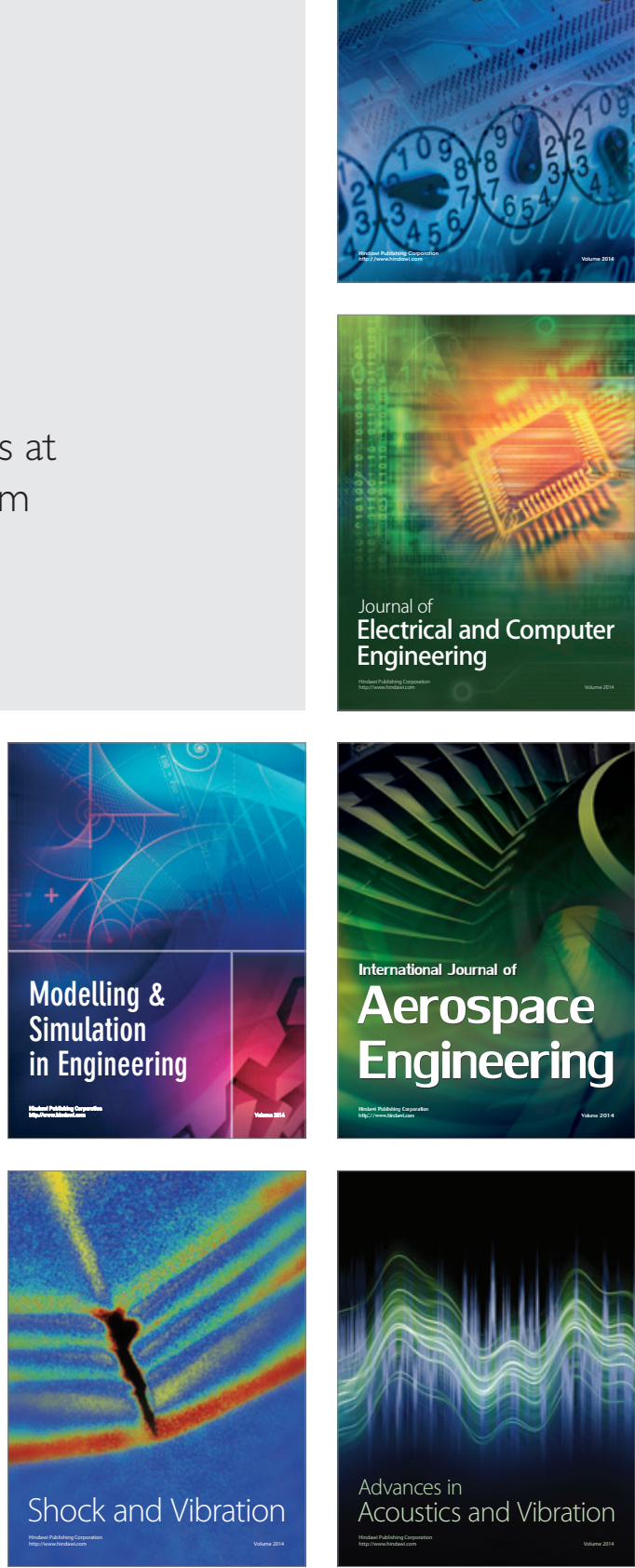